\title{
REVUS
}

Vol. 41 (42) $2020 \bullet$ Art. $1 \bullet$ Especial COVID-19

\section{Hábitos de actividad física y estado de salud durante la pandemia por COVID-19}

\author{
RICO-GALLEGOS, Carina, G. ${ }^{1}$ \\ VARGAS ESPARZA, Goretti ${ }^{1}$ \\ POBLETE-VALDERRAMA, Felipe A. ${ }^{2} 3$ \\ CARRILLO-SÁNCHEZ, José, L. ${ }^{1}$ \\ RICO-GALLEGOS, Jessica. ${ }^{4}$ \\ MENA-QUINTANA , Berenice ${ }^{56}$ \\ CHAPARRO-BAEZA, Diana, K. ${ }^{17}$ \\ RESÉNDIZ-HERNÁNDEZ, Juan, M. ${ }^{8}$
}

Physical activity habits and health status during the pandemic by COVID-19

\begin{abstract}
Resumen
La presente investigación tuvo por objetivo analizar los hábitos de actividad física y estado de salud durante el confinamiento por COVID-19. El diseño del estudio es cuantitativo descriptivo transversal. Se estudió una muestra de 466 personas por medio de una encuesta online, se incluyó el Cuestionario de Actividad Física (IPAQ) y la Escala de Salud de Nottingham para interpretar el comportamiento de los sujetos en términos de actividad física y salud. Los resultados arrojan que $46.35 \%$ realiza actividad física baja, el $21.89 \%$ actividad moderada y un $31.76 \%$ demuestra actividad física de alta intensidad; en términos de estado de salud se demuestran altos niveles de estrés por lo que se puede concluir que es indispensable establecer programas de ejercicio físico para disminuir las consecuencias del sedentarismo en la salud física y mental generado durante el período de confinamiento.
\end{abstract}

Palabras clave: covid-19, actividad física, estilos de vida.

\begin{abstract}
The objective of this investigation was to analyze the physical activity habits and health status during confinement by COVID-19. The study design is quantitative descriptive cross-sectional. A sample of 466 people was studied through an online survey, the Physical Activity Questionnaire was included, and the Nottingham Health Scale were included to interpret the behavior of the subjects in terms of physical activity and health. The results show that $46.35 \%$ perform low physical activity, $21.89 \%$ moderate physical activity and $31.76 \%$ high intensity physical activity, in terms of health status, high levels of stress are demonstrated, so it can be concluded that is essential to establish physical exercise programs to reduce the consequences of sedentary lifestyle on physical and mental health generated during the period of confinement.
\end{abstract}

key words: covid-19, physical activity, lifestyles.

\footnotetext{
${ }^{1}$ Grupo de Investigación Colectivo Palochi, México. cari.ricogallegos@gmail.com, gore.vares@gmail.com , luiscar.96@hotmail.com , menaberenice@gmail.com,diana_chaparro777@hotmail.com

${ }^{2}$ Facultad de Salud, Carrera de Ciencias del deporte y actividad física, Universidad Santo Tomás, Sede Valdivia, Chile. felipepobletev@gmail.com

${ }^{3}$ Facultad de Educación, Universidad Católica de la Santísima Concepción, Chile. felipepobletev@gmail.com

${ }^{4}$ Facultad de Ciencias Políticas y Sociales, Programa de Licenciatura en Relaciones Internacionales, Universidad Autónoma de Querétaro, México. jrico07@alumnos.uaq.mx

${ }^{5}$ Licenciatura en Diseño Industrial, Instituto de Arquitectura Diseño y Arte. Universidad Autónoma de Ciudad Juárez. México. menaberenice@gmail.com

${ }^{6}$ Departamento de Ingeniería industrial. Universidad Tecnológica Paso del Norte. México. menaberenice@gmail.com

7 Programa de Doctorado en Políticas del Bienestar Social, Universidad Autónoma de Nuevo León, México. diana_chaparro777@hotmail.com

${ }^{8}$ Hospital General de Naucalpan Dr. Maximiliano Ruiz Castañeda (ISEM), Estado de México, México.dr.jmresendiz@gmail.com
} 


\section{Introducción}

En diciembre de 2019, apareció un grupo de neumonía con etiología desconocida en la ciudad de Wuhan, provincia de Hubei de China. Varios de los pacientes iniciales visitaron un mercado de mariscos húmedos donde también se vendieron otras especies de vida silvestre. El posterior aislamiento del virus de pacientes humanos y el análisis molecular mostraron que el patógeno era un nuevo coronavirus (CoV), primero denominado 2019nCoV, y posteriormente la Organización Mundial de la Salud (OMS) cambió el nombre de este patógeno como COVID-19 (Gorbalenya, 2020).

Según la OMS (2020) el diagnóstico de la enfermedad se ha realizado como una neumonía inducida por virus con base en los síntomas clínicos que se observaron en los pacientes, similares a los de otros virus respiratorios, a su historia de exposición con otras personas con el virus y la historia de visitas a zonas afectadas. Los pacientes estudiados tenían manifestaciones clínicas de fiebre (83\%), tos (82\%), dificultad para respirar (31\%), dolor muscular (11\%), confusión (9\%), dolor de cabeza (8\%), dolor de garganta (5\%), rinorrea (4\%), dolor en el pecho (2\%), diarrea ( $2 \%)$, náuseas y vómitos (1\%). Según el examen por imágenes, el $75 \%$ de estos pacientes presentaron neumonía bilateral, el $14 \%$ mostró moteado múltiple y opacidad en vidrio esmerilado y el $1 \%$ tenía neumotórax. Además, el 17 \% desarrolló síndrome de dificultad respiratoria aguda y de ellos 11 \% empeoraron en un corto período de tiempo y murieron por insuficiencia orgánica múltiple.

Según informes de los casos ya estudiados, el virus puede afectar a personas de todas las edades, sin embargo, las personas mayores y aquellas que presenten alguna comorbilidad preexistente como diabetes mellitus, asma o enfermedades cardíacas parecen ser más susceptibles a tener mayores complicaciones con el virus. (OMS, 2020)

Las recomendaciones estándar dictadas por la OMS (2020) para prevenir la propagación de la infección incluyen: lavarse las manos regularmente, especialmente después del contacto con personas enfermas o su entorno, cubrirse la boca y la nariz al toser y estornudar, cocinar bien la carne y los huevos, evitar el contacto cercano con cualquier persona que presente síntomas de enfermedades respiratorias (tos y estornudos), evitar viajar a las ciudades y áreas afectadas, así como el tener contacto cercano con animales vivos o muertos de granja o salvajes.

Por otra, parte el Gobierno de México (2020) dictaminó la emergencia sanitaria, por lo que se acordó la suspensión de actividades no esenciales hasta el día 30 de mayo en algunos estados y municipios donde hay mayor concentración de casos, con la finalidad de mitigar los casos de contagio por COVID-19, por lo cual una gran población permanece en sus hogares, las escuelas en todos los niveles educativos se encuentran dando las clases en línea, la mayoría de los espacios públicos se encuentran cerrados, obligando así a la población a hacer caso al comunicado oficial de quedarse en casa.

\subsection{Actividad y ejercicio físico durante el confinamiento por COVID-19}

Las restricciones obligatorias sobre la participación en actividades al aire libre, incluida la actividad física regular y el ejercicio, interrumpen inevitablemente las actividades cotidianas de decenas de millones de personas. La Organización Mundial de la Salud (2020) establece que las personas tienen la oportunidad de mantenerse físicamente activas en cuatro sectores principales de la vida diaria: el trabajo, el transporte, las tareas domésticas y el tiempo libre o de ocio; recomendando utilizar instrumentos de medición capaces de recoger información en todas estas dimensiones. Entre los instrumentos existentes para medir la actividad física se puede mencionar el Cuestionario Internacional de Actividad Física (IPAQ).

EI IPAQ consta de 7 preguntas, donde se evalúan 3 características de la actividad física: intensidad (leve, moderada o intensa), frecuencia (días por semana) y duración (tiempo por día). Se debe calcular el valor semanal de actividad física a través de METs-min-semana, (MET, Unidad de medida del índice metabólico) donde se 
consideran los valores de referencia de caminar (3.3 METs), actividad física moderada (4 METs), y actividad física vigorosa (8 METs). El test se puede aplicar mediante entrevista directa, vía telefónica o encuesta auto cumplimentada.

Dada la situación que actualmente se está viviendo a consecuencia de la pandemia por COVID-19, la realización de las actividades físicas ha disminuido considerablemente a pesar de las recomendaciones que varias instituciones están poniendo a disposición del público; una de las recomendaciones que establece la OMS es mantener una rutina para mantenerse activo todos los días, con ejercicios que se puedan realizar estando solos, o realizar una clase por internet, un encuentro en línea con amigos para hacer juntos ejercicio, que de alguna manera se cumpla para crear una rutina y adaptarse a las nuevas formas de trabajo, estudio y vida familiar en marco de las restricciones impuestas durante la pandemia por COVID-19 (OMS, 2020).

Un estudio realizado por Chen y colaboradores (2020) hace referencia que hacer actividad en casa usando varios ejercicios seguros y de fácil implementación, es muy adecuado para evitar la exposición a contagio de coronavirus y mantener los niveles de condición física. Dichas formas de ejercicio pueden incluir, entre otras, ejercicios de fortalecimiento, actividades de equilibrio, ejercicios de estiramiento o una combinación de éstos. Los ejemplos de ejercicios en el hogar incluyen caminar en la casa, levantar y cargar alimentos, alternar estocadas de piernas, subir escaleras, ponerse de pie y sentarse usando una silla, sentadillas, y abdominales. Recomiendan, además, Tai Chi y yoga ya que no requieren equipo, se usa poco espacio y se pueden practicar en cualquier momento. El uso de videos por Internet con temáticas de ejercicios, que se enfocan en alentar y realizar actividad física a través de tecnologías móviles y televisión, son otras vías viables para mantener la función física y la salud mental durante este período crítico.

Diversas instituciones proponen ajustar las recomendaciones internacionales sobre actividad física a la situación actual (American College of Sports Medicine, Sociedad Española de Medicina del Deporte, Organización Mundial de la Salud, Exercise and Sport Sciences Australia, 2020). En general, todas las instancias proporcionan las mismas recomendaciones, permanecer activo en casa, hacer pausas activas cortas y evitar los periodos de sedentarismo excesivo.

Los aparatos portátiles para la salud han hecho que nunca haya sido más fácil para los usuarios sin conocimientos previos cuantificar y controlar las rutinas de ejercicio. Sin embargo, debido a la amplia variedad de plataformas dedicadas al fomento del ejercicio físico, es necesario consultar canales informativos creados por las sociedades científicas, los médicos y los profesionales la salud para conseguir un estado físico cardiovascular y osteomuscular óptimo, dentro de los límites permitidos por la cuarentena. (Rodríguez, 2020).

El contexto ha permitido además desarrollar una serie de intervenciones mediante todas las plataformas digitales y redes sociales, poniendo a disposición múltiples métodos de acceso a nuevos aprendizajes, ya sea como se ha mencionado rutinas de ejercicio físico, pero además la población se ha permitido desarrollar nuevas habilidades o intereses; tales como aprender a cocinar, aprender a tocar un instrumento emprender un negocio.

Según Vázquez (2020) una de las industrias que se mantienen en pie e incluso se vieron beneficiadas es el sector del fitness. Si bien comenzaba a explotar el mercado desde hace tiempo, la pandemia llegó a acelerar los procesos. Mientras Nike dio acceso gratuito a su servicio premium de rutinas de ejercicio, a inicios de marzo, MacRumors reportó que Apple trabajaba en una nueva aplicación para realizar ejercicio.

De diciembre a marzo el porcentaje de horas en aplicaciones android relacionadas a la salud, servicio médico y fitness incrementaron principalmente en Estados Unidos (30\%), China (20\%), Japón (20\%) y Francia (15\%), indican datos de App Annie, compañía experta en el análisis de datos móviles. 
En los supermercados en México, además de la alta demanda en desinfectante, los artículos de fitness también han llegado a agotarse. La firma de estudios Alpha indicó que el home fitness mueve 14,000 millones de dólares a nivel mundial. (Vázquez, 2020)

\subsection{Percepción de la salud en tiempos de pandemia}

Las repercusiones en cuestiones de salud son múltiples y muy variadas en opinión de las personas en este proceso de adaptación que se ha tenido que incorporar e integrar en las formas de vida de muchas personas, todo un conjunto de hábitos no saludables en el ejercicio, alimentación, en el descanso, en el conjunto de sus rutinas cotidianas que permiten caracterizar el "estilo de vida no saludable" durante el confinamiento por COVID-19.

La Iner-Agency Standing Committee IASC (2010) menciona que es necesario enfatizar en la perspectiva conductual y lograr una mejor comprensión de las respuestas de los grupos poblacionales afectados. El control de las epidemias requiere cambios de patrones de comportamiento, con una participación activa de la comunidad. Muchos de los problemas que afligen a las personas en situaciones de epidemias, tienen su origen en el miedo en sus múltiples expresiones. Estudios realizados han identificado que hasta más del $80 \%$ de las personas, en circunstancias de cercanía evidente al peligro, expresan manifestaciones sintomáticas de angustia e incluso pánico.

La Escala de Salud Nottingham (NHP) es una encuesta empleada para medir la percepción subjetiva del impacto de los problemas de salud; es un instrumento que mide el dolor físico, psicológico y social asociado a problemas médicos, sociales y emocionales. Siendo un instrumento que ha sido de gran utilidad en este estudio al permitir la visualización de manera más objetiva de la situación en cuestiones de salud que las personas están llevando en este momento. (Medina, 2013)

\subsection{Objetivo}

El propósito del presente estudio es analizar los hábitos de actividad física y estado de salud en la población mexicana durante el confinamiento por COVID-19.

\section{Metodología}

\subsection{Material y métodos}

Estudio cuantitativo transversal y descriptivo, realizado a través de una encuesta On-line utilizando la aplicación de Google Docs distribuida en México en el período de marzo-abril 2020. Se invitó a participar a una muestra de la población mexicana a través del envío de un enlace a través de la Web $(n=466)$. Se incluyeron sujetos que accedieron a brindar su consentimiento informado y cooperaron en las evaluaciones del estudio.

La muestra total fue de 466 sujetos; 328 femeninos y 136 masculinos, con un promedio de edad de 32,77 $\pm 12,28$. Se realizó el Cuestionario de Actividad Física (IPAQ) y la Escala de Salud de Nottingham. Los resultados descriptivos se expresaron en porcentajes para el total de la muestra, según el género y rango de edad, así como medidas de tendencia central de acuerdo al nivel de actividad física y el estado de percepción de salud. Los datos fueron registrados en plantillas diseñas para este estudio y empleando el programa SPSS.

\subsection{Evaluaciones}

Cuestionario de Actividad Física (IPAQ): Calcula el nivel de actividad física a través de preguntas en cuatro dominios: laboral, doméstico, de transporte y del tiempo libre. El indicador de actividad física se expresa tanto de manera continua, en MET-minutos/semana, como de manera categórica, clasificando el nivel de actividad física en bajo, moderado o alto. (Lee, 2011) 
Escala de Salud de Nottingham: Evalúa el impacto de la enfermedad sobre la percepción de salud del individuo. Consta de dos partes: la primera comprende 38 ítems agrupables en seis dimensiones: energía, dolor, movilidad, reacciones emocionales, sueño y aislamiento social. La segunda parte incluye siete preguntas sobre la existencia de problemas debidos al estado de salud en diferentes aspectos: trabajo, tareas domésticas, vida social, vida familiar, vida sexual, aficiones y períodos de ocio. Todas las preguntas, en ambas partes, se responden "Sí» o «No». Se puede puntuar por el porcentaje de respuestas afirmativas para cada dimensión (respuestas positivas/número de ítems, multiplicado por 100). La puntuación de las dimensiones ofrece un perfil del estado de salud percibida por el sujeto. (Medina, 2013)

\section{Resultados}

A continuación, se presentan los resultados obtenidos tras la aplicación de los test.

Tabla 1

Características de la muestra

\begin{tabular}{|c|c|c|c|c|}
\hline Variable & \multicolumn{4}{|c|}{ Cantidad de sujetos $(n=466)$} \\
\hline Femenino & \multicolumn{4}{|c|}{328} \\
\hline Masculino & \multicolumn{4}{|c|}{136} \\
\hline & Promedio & Desv. Est & Mínimo & Máximo \\
\hline Edad & 32,77 & 12,28 & 14 & 81 \\
\hline
\end{tabular}

La tabla 1, Características de la muestra, expone las medidas de tendencia central del estudio para la variable de edad y distribución de acuerdo al género, donde se observa un 70,4 \% ( $\mathrm{n:328)}$ correspondiente al género femenino y $29,6 \%$ (n:136) género masculino. En la variable edad no existió criterio de exclusión, se obtuvo un promedio de $32,77 \pm 12,28$.

Tabla 2

Prevalencia de nivel de actividad física según género y rango de edad

\begin{tabular}{|c|c|c|c|c|c|c|c|}
\hline \multirow{2}{*}{$\begin{array}{c}\text { Nivel de } \\
\begin{array}{c}\text { actividad } \\
\text { física }\end{array}\end{array}$} & $\begin{array}{c}\text { Prevalencia } \\
\text { total (\%) } \\
(n=466)\end{array}$ & $\begin{array}{c}\text { Femenino } \\
(n=328)\end{array}$ & $\begin{array}{c}\text { Masculino } \\
(n=138)\end{array}$ & $\begin{array}{c}0-20 \\
\text { años } \\
(n=20)\end{array}$ & $\begin{array}{c}21-40 \\
\text { años } \\
(n=337)\end{array}$ & $\begin{array}{c}41-60 \\
\text { años } \\
(n=93)\end{array}$ & $\begin{array}{c}\text { Género (\%) } \\
\text { años } \\
(n=16)\end{array}$ \\
\hline Bajo & $46,35 \%$ & $49,39 \%$ & $39,13 \%$ & $25 \%$ & $44,21 \%$ & $59,13 \%$ & $50 \%$ \\
\hline Moderado & $21,89 \%$ & $21,34 \%$ & $23,18 \%$ & $15 \%$ & $23,73 \%$ & $17,20 \%$ & $12,5 \%$ \\
\hline Alto & $31,76 \%$ & $29,26 \%$ & $37,68 \%$ & $60 \%$ & $32,04 \%$ & $23,65 \%$ & $37,5 \%$ \\
\hline
\end{tabular}

La tabla 2, Prevalencia de nivel de actividad física según el género y rango de edad, expone los porcentajes de la población de acuerdo al nivel de actividad física que realizan; con mayor predominio del nivel bajo con 46,35\% ( $n=217)$, seguido del nivel alto $31,76 \%(n=148)$, y finalmente el moderado $21,89 \%(n=101)$. Por otra parte, la frecuencia de los diferentes niveles de actividad física fue significativamente distinta con respecto al género, donde se muestra en el femenino mayor prevalencia en el nivel bajo con respecto al masculino.

En la distribución por grupo de edad, se observa mayor frecuencia del nivel bajo de actividad física en todos los rangos de edad, excepto en el de 0-20 años, en donde predomina el nivel alto. 
Tabla 3

Resultados del cuestionario de salud de Nottingham

\begin{tabular}{|c|c|c|}
\hline Dimensiones & $\begin{array}{l}\text { Cantidad de sujetos con } \\
\text { respuesta afirmativa }\end{array}$ & $\begin{array}{l}\text { Porcentaje de respuestas } \\
\text { afirmativas (\%) }\end{array}$ \\
\hline \multicolumn{3}{|l|}{ Energía } \\
\hline Los días se me hacen interminables & 110 & $23,60 \%$ \\
\hline Siempre estoy fatigado & 78 & $16,73 \%$ \\
\hline Todo me cuesta esfuerzo & 26 & $5,57 \%$ \\
\hline Enseguida me quedo sin fuerzas & 24 & $5,15 \%$ \\
\hline \multicolumn{3}{|l|}{ Dolor } \\
\hline Dolor por las noches & 69 & $14,80 \%$ \\
\hline Dolor insoportable & 21 & $4,50 \%$ \\
\hline Dolor al cambiar de posición & 118 & $25,32 \%$ \\
\hline Dolor al estar de pie & 47 & $10,08 \%$ \\
\hline Dolor constante & 46 & $9,87 \%$ \\
\hline Dolor al subir y bajar escaleras & 31 & $6,65 \%$ \\
\hline Dolor cuando estoy sentado & 59 & $12,66 \%$ \\
\hline \multicolumn{3}{|l|}{ Reacciones emocionales } \\
\hline Preocupaciones me desvelan & 127 & $27,25 \%$ \\
\hline Últimamente me enfado con facilidad & 196 & $42,06 \%$ \\
\hline Las cosas me deprimen & 138 & $29,61 \%$ \\
\hline He olvidado lo que es pasarla bien & 57 & $12,23 \%$ \\
\hline Tengo los nervios de punta & 102 & $21,88 \%$ \\
\hline Creo que soy una carga para los demás & 30 & $6,43 \%$ \\
\hline $\begin{array}{l}\text { Siento que estoy perdiendo el control de mí } \\
\text { mismo }\end{array}$ & 39 & $8,36 \%$ \\
\hline Creo que no vale la pena vivir & 22 & $4,72 \%$ \\
\hline Me despierto desanimado & 91 & $19,52 \%$ \\
\hline \multicolumn{3}{|l|}{ Sueño } \\
\hline Tomo pastillas para dormir & 20 & $4,29 \%$ \\
\hline Me despierto antes de la hora & 172 & $36,90 \%$ \\
\hline $\begin{array}{l}\text { Me la paso despierto la mayor parte de la } \\
\text { noche }\end{array}$ & 95 & $20,38 \%$ \\
\hline Me cuesta mucho dormir & 146 & $31,33 \%$ \\
\hline Duermo mal por las noches & 130 & $27,89 \%$ \\
\hline \multicolumn{3}{|l|}{ Aislamiento social } \\
\hline Me cuesta llevarme bien con la gente & 39 & $8,36 \%$ \\
\hline $\begin{array}{l}\text { Últimamente me resulta difícil contactar con } \\
\text { la gente }\end{array}$ & 63 & $13,51 \%$ \\
\hline Me encuentro solo & 55 & $11,80 \%$ \\
\hline Creo que no tengo a nadie en quien confiar & 33 & $7,08 \%$ \\
\hline \multicolumn{3}{|l|}{ Movilidad física } \\
\hline Sólo puedo andar dentro de la casa & 164 & $35,19 \%$ \\
\hline Me cuesta coger las cosas & 10 & $2,14 \%$ \\
\hline
\end{tabular}




\begin{tabular}{|l|c|c|}
\hline \multicolumn{1}{|c|}{ Dimensiones } & $\begin{array}{c}\text { Cantidad de sujetos con } \\
\text { respuesta afirmativa }\end{array}$ & $\begin{array}{c}\text { Porcentaje de respuestas } \\
\text { afirmativas (\%) }\end{array}$ \\
\hline Me cuesta agacharme & 52 & $11,15 \%$ \\
\hline Incapacidad de andar & 4 & $0,85 \%$ \\
\hline Subir y bajar escaleras & 36 & $7,72 \%$ \\
\hline Me cuesta vestirme & 15 & $3,21 \%$ \\
\hline $\begin{array}{l}\text { Necesito ayuda para trasladarme (silla de } \\
\text { ruedas, bastón, muletas) }\end{array}$ & 2 & $0,42 \%$ \\
\hline $\begin{array}{l}\text { Me cuesta trabajo mantenerme mucho } \\
\text { tiempo de pie }\end{array}$ & 56 & $12,01 \%$ \\
\hline Limitación a causa de salud & 36 & $7,72 \%$ \\
\hline Actividades domésticas & 34 & $7,29 \%$ \\
\hline Familiar & 39 & $8,36 \%$ \\
\hline Sexual & 55 & $11,80 \%$ \\
\hline Pasatiempos & 56 & $12,01 \%$ \\
\hline Social & & \\
\hline
\end{tabular}

La tabla III, Resultados del Cuestionario de Salud de Nottingham, representa la cantidad de sujetos que respondieron de manera afirmativa a cada ítem, así como sus respectivos porcentajes, donde se observa mayor proporción en la dimensión de reacciones emocionales, seguido de alteraciones en el sueño. En los parámetros de limitación en el estado de salud, destaca el ítem social con 12,01 \% ( $n=56)$, asociado al aislamiento por el período de la contingencia.

El Cuestionario de Salud de Nottingham ofrece un perfil del estado de salud percibida por el sujeto, donde se obtiene una cifra máxima de $100 \%$.

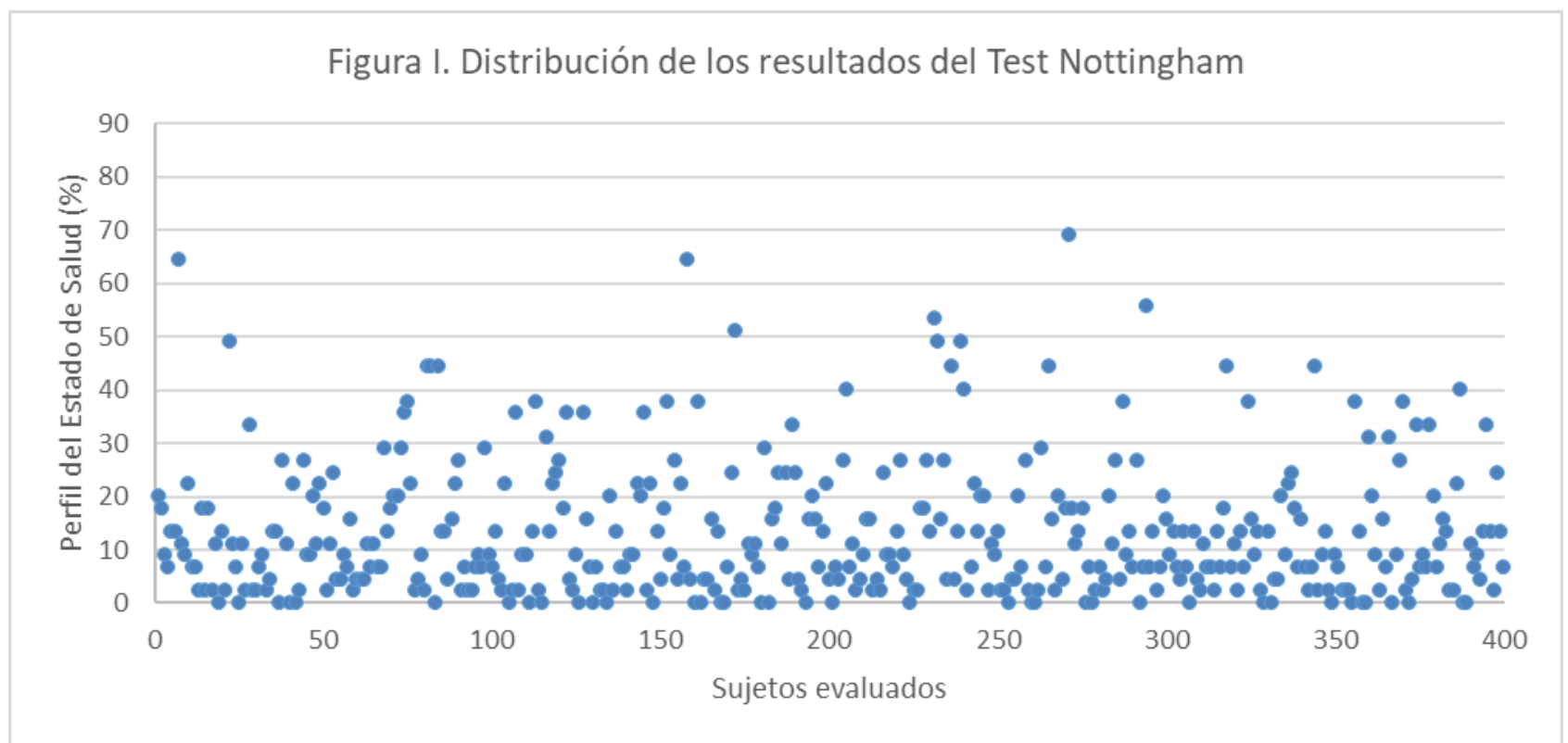

En la figura I, se muestra la distribución de los resultados, donde se obtuvo un promedio de $13,23 \%$, con una desviación estándar de 13,48, una cifra mínima de 0 \% y máxima de $84,4 \%$. 


\subsection{Discusiones}

Los principales hallazgos estuvieron enmarcados en los estilos de vida debido al confinamiento por la pandemia ocasionada por COVID-19, lo cual ha generado un aumento y/o prevalencia de patrones de conducta sedentaria. El efecto que ha causado en la población demuestra que un gran porcentaje de las personas encuestadas presenta alteraciones en el sueño, falta de motivación al realizar sus actividades de la vida diaria y con tendencia al estrés. Estos resultados son relevantes ya que comprueban que el sedentarismo y aislamiento social tiene repercusiones a nivel de estado físico y emocional.

Si bien, actualmente no existen estudios que hagan evidencia respecto a estilos de vida durante un período tan largo de confinamiento, este estudio concuerda con los estudios realizados por (Brooks et al, 2004) donde concluyen que si es cierto que el aislamiento es importante para proteger nuestra salud física y prevenir la infección por el virus, también es cierto que cuanto más tiempo estemos aislados, mayores serán los riesgos de sufrir enfermedades psiquiátricas. Sabemos que la cuarentena puede causar una constelación de síntomas psicopatológicos, afectación en el estado de ánimo, depresión, irritabilidad, ansiedad, miedo, ira, insomnio, etcétera. Además, en otros estudios de cuarentenas no tan largas como ésta, se han identificado consecuencias a largo plazo para la salud mental aproximadamente tres años después de la cuarentena, donde hubo un mayor riesgo de aparición de abuso de alcohol, síntomas de trastorno de estrés postraumático y depresión (Wu, Liu, Fang, Fan, Fuller y Guan, 2008).

Por otra parte, Chen, Mao, Nassis, Harmer, Ainsworth y Li (2020) hacen mención acerca de un estudio reciente, donde se señala que las medidas oficiales que restringen los movimientos de las personas en presencia de la crisis del coronavirus no necesariamente significan que la actividad física debe ser limitada o que todas las formas de ejercicio deben eliminarse por completo. Se ha demostrado que el ejercicio tiene claros beneficios para la salud de individuos sanos y para pacientes con diversas enfermedades, además concluye que la estadía en el hogar es un paso fundamental de seguridad que puede limitar la propagación de infecciones. Pero las estadías prolongadas en el hogar pueden aumentar los comportamientos que conducen a la inactividad y contribuyen a la ansiedad y la depresión, lo que a su vez puede conducir a un estilo de vida sedentario que llevaría a una variedad de condiciones de salud crónicas. Por lo tanto, mantener una actividad física regular y hacer ejercicio de manera rutinaria en un ambiente hogareño seguro es una estrategia importante para una vida saludable durante la crisis del coronavirus.

Actualmente se encuentran en desarrollo más alternativas para mantener en adecuadas condiciones los niveles de actividad física y salud, diseñados para realizarse en casa. Desde guías diseñadas por instituciones del ámbito de la actividad física y deporte o cuidado de la salud. Además, que se están sumando diversos profesionales de la salud para aportar educación sobre el cuidado de sí a la población en general. Algunos profesionales se suman a las clases de ejercicio por medio de plataformas como ZOOM, MEET, trasmisiones en directo por Facebook, Instagram y otras redes sociales. Lo cual ha generado un impacto en la industria del Fitness y al mismo tiempo un efecto en los estados físicos y emocionales de las personas que se han sumado a estas alternativas.

\section{Conclusiones}

Describir las variables de actividad física y estado de salud en general en la población que actualmente se encuentra bajo las restricciones sociales, académicas y laborales debido a la contingencia a consecuencia de la pandemia por COVID-19, permite determinar que según el Cuestionario Internacional de Actividad Física (IPAQ) un $46.35 \%$ realiza una actividad física baja, el $21.89 \%$ una actividad moderada y un 31.76 \% demuestra una actividad física de alta intensidad. En relación a el estado de salud, valorado con la Escala de Salud de Nottingham se encuentran impactos en el área emocional, principalmente en el estado de ánimo y en el sueño (cabe señalar 
aquí lo benéfico de potenciar la realización de actividades físicas en el hogar, lo cual traería mejoras de índole emocional).

Si bien, comprender y contener el virus es prioridad de salud pública. Estos hechos sugieren que es importante incorporar medidas de información y acciones ante estos posibles eventos (pandemias y otros problemas de salud pública) que han ocurrido a lo largo de la historia y muy probablemente volverán a ocurrir en un futuro, para así evitar en medida de lo posible un cambio rotundo de los estilos de vida, ya que no se le ha dado la importancia suficiente a lo que la población en general debería hacer en cuestiones de ejercicio y actividad física. Podría decirse que quedarse en casa, para evitar la transmisión del virus de persona a persona, aunque sea una medida segura, puede tener consecuencias no deseadas, ya que tales esfuerzos pueden conducir a una menor actividad física.

Es muy probable que la estadía prolongada en los hogares conduzca a un aumento de las conductas sedentarias, como pasar demasiado tiempo sentado o acostado para realizar actividades de ocio (video juegos, ver televisión, usar dispositivos móviles, leer) generando una disminución considerable de las actividades físicas regulares.

Es de suma importancia seguir generando investigaciones con respecto al tema de confinamiento, patrones de vida sedentaria y sus posibles consecuencias en un futuro cercano, los profesionales que se dedican a la promoción de estilos de vida saludable son indispensables en estos momentos, generando una participación activa de la población al realizar de manera frecuente ejercicio físico, no sólo ahora, ni pasando el confinamiento solamente, sino que ello se vuelva parte de la rutina diaria. Estas medidas preventivas apoyarán a que en una próxima contingencia o emergencia sanitaria no se repitan estos patrones y cambiemos el contexto de que confinamiento es sinónimo de enfermedad física y/o mental.

\section{Referencias bibliográficas}

American College of Sports Medicine. Exercises Medicine. Disponible en: https://www.exerciseismedicine.org/support_page.php/stories/?b=892. Consultado 22 Ago 2020.

Brooks, S., Webster, R., Smith, L., Woodland, L., Wessely, S., Greenberg, N. y Gideon, J. (2020). The psychological impact of quarantine and how to reduce it: rapid review of the evidence. The Lancet, 395, 912-920.

Chen, P., Mao, L., Nassis, G. P., Harmer, P., Ainsworth, B. E., y Li, F. (2020). Wuhan coronavirus (2019-nCoV): The need to maintain regular physical activity while taking precautions. Journal of sport and health science, 9 (2), 103-104.

Cruz, M. P., Santos, E., Cervantes, M. V., y Juárez, M. L. (2020). COVID-19, una emergencia de salud pública mundial. Revista Clínica Española. Recuperado de https://www.ncbi.nlm.nih.gov/pmc/articles/PMC7102523/

Gobierno de México. (2020). Coronavirus. Abril 2020, de Gobierno de México Sitio web: https://coronavirus.gob.mx/

Gorbalenya, A. E. (2020). Severe acute respiratory syndrome-related coronavirus-The species and its viruses, a statement of the Coronavirus Study Group. BioRxiv. Recuperado de https://www.biorxiv.org/content/10.1101/2020.02.07.937862v1

Fundación Española del Corazón. Pautas para hacer ejercicio en casa durante la situación de emergencia por el coronavirus. Disponible en: https://fundaciondelcorazon.com/blog-impulso-vital/3513-pautas-para-hacerejercicio-en-casa-durante-la-situacion-de-emergencia-por-el-coronavirus.html. Consultado 22 Ago 2020. 
Hawryluck, L., Gold, W. L., Robinson, S., Pogorski, S., Galea, S. y Styra, R. (2004). SARS control and psychological effects of quarantine, Toronto, Canada. Emerging Infectious Diseases, 10 (7), 1206-1212.

Iner-Agency Standing Committee IASC (2010). Reference Group for Mental Health and Psychosocial Support in Emergency Settings. Mental Health and Psychosocial Support in Humanitarian Emergencies: ¿What Should Humanitarian Health Actors Know? Geneva. Recuperado en:

http://www.who.int/mental_health/emergencies/what_humanitarian_health_actors_should_k now.pdf

Lee, P. H., Macfarlane, D. J., Lam, T. H., \& Stewart, S. M. (2011). Validity of the International Physical Activity Questionnaire Short Form (IPAQ-SF): A systematic review. The International Journal of Behavioral Nutrition and Physical Activity, 8, Article 115

Medina C, Barquera S, Janssen I. (2013) Validity and reliability of the International Physical Activity Questionnaire among adults in Mexico. Rev Panam Salud Publica. ;34 (1): 21-8.

Vázquez, F.. (2020). Home Fitness, más demanda para las apps. 22 Ago 2020, de El economista Sitio web: https://www.eleconomista.com.mx/deportes/Home-Fitness-mas-demanda-para-las-apps-202003260148.html

Organización Mundial de la Salud (2020). Coronavirus. Recuperado en: https://www.who.int/es/emergencies/diseases/novel-coronavirus-2019

World Health Organization. Stay physically active during self-quarantine. Disponible en: http://www.euro.who.int/en/health-topics/health-emergencies/coronavirus-covid-19/novel-coronavirus2019-ncov-technical-guidance/stay-physically-active-during-self Consultado: 22 Ago 2020.

Wu, P., Liu, X., Fang, Y., Fan, B., Fuller, C. y Guan, Z. (2008). Alcohol abuse/dependence symptoms among hospital employees exposed to a SARS outbreak. Alcohol \& Alcoholism, 43 (6), 706-712.

Esta obra está bajo una Licencia Creative Commons Attribución-NoCommercial 4.0 International

(cc) BY-NC 